\title{
Brain-Derived Neurotrophic Factor-Major Depressive Disorder and Suicide
}

\author{
Roberto Galea1,2*, David Cassar² \\ ${ }^{1}$ Mount Carmel Hospital, Attard, Malta \\ ${ }^{2}$ Department of Psychiatry, Faculty of Medicine and Surgery, University of Malta, Imsida, Malta \\ Email: *galea.roberto@gmail.com
}

How to cite this paper: Galea, R. and Cassar, D. (2019) Brain-Derived Neurotrophic Factor-Major Depressive Disorder and Suicide. Open Access Library Journal, 6: e5106.

https://doi.org/10.4236/oalib.1105106

Received: December 11, 2018

Accepted: January 13, 2019

Published: January 16, 2019

Copyright $\odot 2019$ by author(s) and Open Access Library Inc.

This work is licensed under the Creative Commons Attribution International License (CC BY 4.0).

http://creativecommons.org/licenses/by/4.0/

\begin{abstract}
Objectives: The aims and objectives of this systematic review are to investigate the different theories underlining major depression and suicide while exploring the correlation between BDNF, major depression and suicide. Method: Literature was researched through the University of Malta research platform and statistical reports published by the Maltese National Statistics Office, Eurostat and World Health Organisation were consulted. Depression: Low blood BDNF levels have been identified to occur in major depression which normalise during remission. Additionally, since BDNF is associated with increased neural plasticity and survival and decreased atrophy, exposure to chronic stress was identified to decrease BDNF levels and reverse such traits in laboratory rats. Single nucleotide polymorphism Val66Met on the BDNF gene has been identified to be responsible for decreased activity-dependent secretion of BDNF and resultantly increased depressive features. Sertraline administration in human subjects and laboratory rats has been also identified to have a neurogenic effect on serotonergic and BDNF neurons; increasing their expression. Suicide: Despite that suicide is commonly associated as part of the symptomatology of psychiatric disorders, in light that children of suicide attempters are six times more susceptible to attempt suicide than children of non-suicide attempters; recent research considered suicide as an independent phenomenon with a high percentage of heritability. Higher concentrations of cholecystokinin receptors and cholecystokinin B receptors have been reported in individuals who died by suicide. Conclusion: Large randomised replicated research looking into BDNF, major depression and suicide is still absent. Further research is necessary to validate the reported findings.
\end{abstract}

\section{Subject Areas}

Drugs \& Devices, Epidemiology, Neuroscience, Psychiatry \& Psychology 


\section{Keywords}

BDNF, Major Depressive Disorder, Depression, Suicide and Suicide Attempts

\section{Introduction}

Major Depressive Disorder (MDD) is the commonest major psychiatric illness globally with an estimated 350 million effected individuals across all age groups [1]. MDD is the major cause of disability and is the major contributor to the overall burden of disease globally (Davies \& Craig, 2009; Abramets et al., 2015; WHO, 2016). Untreated depressive episodes may last between six to 24 months with individuals experiencing first-onset episodes exhibiting 50\% - 60\% chance of experiencing a second one. Additionally, around $9 \%$ of those diagnosed with MDD also described psychotic features [2] [3]. Depression is strongly correlated to suicide; $80 \%$ of individuals who commit suicide suffer from depression while $15 \%$ of those diagnose with MDD commit suicide [4].

Suicide is a major cause of death within the European Union (EU); in 2011, 60,000 individuals committed suicide [5]. Statistically suicide accounts for $17.6 \%$ of all deaths among young adults in high-income countries, ranking as the second leading cause of death within this age group following road traffic accidents both in the EU and globally [6] [7]. Suicide is the second leading cause of death amongst 15 - 29-year olds [1]. In the United Kingdom, 5000 individuals die by suicide each year and it is estimated that $\sim 450$ involve overdose with antidepressants [2]. Malta has a rate of 5.1 suicides per 100,000 inhabitants [8].

\section{Pathophysiological Theories of Depression}

\subsection{Monoamine Hypothesis}

The monoamine hypothesis describes that MDD is a result of a dysfunctional monoamine neurotransmitter system, particularly; serotonin, noradrenaline, adrenaline and dopamine, resulting in decreased extracellular monoamines concentrations and neurotransmission (Ruhe et al., 2007; Jentsch et al., 2015). The decrease in monoamine neurotransmission possibly results from three factors; too little neurotransmitters released by presynaptic neurons, rapidly reabsorbed/degraded neurotransmitters or a decrease in postsynaptic receptors [2]. Decreased monoamine metabolite concentrations have been also identified in the Cerebrospinal Fluid (CSF), blood, urine and post-mortem brain tissue of individuals diagnosed with MDD (Jentsch et al., 2015). Research looking into this pathophysiological theory mimicked monoamine neurotransmitter depletion by tryptophan, phenylalanine and tyrosine depletion (precursors) by removing them from the diet or blocking their synthesis; this "depletion" induced depressive symptoms. Depressive symptoms were noticed in individuals with a history of MDD and non-depressed relatives of MDD individuals but not in healthy individuals without a personal/familial history of MDD (Andrews et al., 2015; 
Jentsch et al., 2015). Females scored higher on depressive index scales than males across all the recruited groups. A clear summary of the effects monoamine depletion has on mood across different populations is absent while inconclusive data lead to deduct that changes in the monoamine system is only a partial explanation of the aetiology of MDD (Ruhe et al., 2007).

\subsection{Hypothalamic-Pituitary-Adrenal Axis}

Activation of neuroendocrine systems is a primary physiological response to stress, principally by the Hypothalamic-Pituitary-Adrenal (HPA) axis. Emotional stimuli reach the amygdala and forebrain which stimulate the HPA axis. The amygdala stimulates the hypothalamus which in turn excites the HPA axis which by increasing blood cortisol concentrations acts on a positive feedback mechanism to further stimulate the amygdala. Over-excitation of the HPA axis is inhibited with a negative feedback mechanism by the hippocampus which limits the HPA axis activity (Willner et al., 2013); dysregulation of the HPA axis has been implicated in the aetiology of MDD. Research identified elevated cortisol, adrenocorticotrophic hormone and corticotropin releasing hormone levels in blood, urine, saliva, CSF and post-mortem brain tissue of individuals diagnosed with MDD (Jentsch et al., 2015). Elevated glucocorticoid concentrations have been identified to cause inhibition of medial prefrontal cortex excitation in MDD (Abramets et al., 2015). However only part of the individuals diagnosed with MDD exhibit HPA axis abnormalities. Thus, these conclusions may only be a feature of specific subtypes of depression (Jentsch et al., 2015).

\subsection{Inflammatory Hypothesis}

The inflammatory hypothesis suggests that inflammatory changes, primarily; increased pro-inflammatory cytokine, decreased anti-inflammatory cytokine concentrations and activation of immune cells to be partially related to the onset of MDD. Supporting such hypothesis are increased levels of interleukin-1, interleukin- 6 and decreased levels of anti-inflammatory cytokines (interleukin- 4 and interleukin-10), recognising pro-inflammatory markers to cause depressive-like symptoms. Pro-inflammatory cytokines are also activated in other disorders not necessarily related to depression and not causing depression; eliminating the inflammatory hypothesis as a prime causative factor of MDD (Jentsch et al., 2015).

\subsection{Treatment}

Antidepressant treatment for MDD includes; cyclic antidepressants, monoamine oxidase inhibitors, Selective Serotonin Reuptake Inhibitors (SSRIs) and atypical antidepressants. Increased neurotransmission is achieved either through increased serotonin, noradrenaline and dopamine synaptic concentrations or by agonistic effect on the respective receptors (Ruhe et al., 2007). Electroconvulsive therapy (ECT) is the last line treatment for treatment resistant individuals or those experiencing severe side-effects. During ECT an electric impulse is passed through the brain causing a seizure which is believed to correct the brain's 
chemical imbalance in MDD [2].

\section{Neurotrophins}

Neurotrophins (NTs) are a group of proteins part of the neurotrophic factors family. NTs are found both in the central and peripheral nervous system and provide for neural; survival or death, growth or retraction and synaptic stabilisation or elimination mediated through NT specific membrane bound receptors (Wang et al., 2012). Nerve Growth Factor (NGF) was the first NT identified. Samples containing proteins secreted by cultured neuronal and non-neuronal cells also supported the survival of Non-NGF dependant neurons and that did not express NGF receptors; suggesting the existence of additional NTs (Squire et al., 2008). Different NTs effect specific types of neurons. NGF supports sympathetic neurons, BDNF supports sensory ganglion neurons while NT-3 supports both populations. NT action is mediated through two classes of receptors; Tyrosine kinase (Trk) and p75 receptors which exhibit specificity for different NTs (TrkA-NGF, TrkB-BDNF and NT-4/5 and TrkC-NT-3), however all NTs can activate the p75 receptor (Fukui et al., 2010; Qian et al., 2010).

Signalling through either Trk or p75 receptors determines the resultant outcomes sensitive to NTs signalling through different signalling cascades which alter the cell status and gene expression. The outcome of NTs interaction is thus determined by; local NT availability, neuronal receptors and intracellular signalling pathways expressed (Cunha et al., 2010). The disruption of NT-dependant processes can possibly cause neurodegenerative conditions due to inappropriate trophic support and the failure to make and maintain neural connections. The neurotrophic hypothesis suggests that NTs are secreted from postsynaptic neurons and bind to presynaptic neurons for internalisation, retrograde axonal transport and signal transduction. However, NT receptors are also found on postsynaptic neurons. Research shows that NTs have rapid effects on synaptic transmission with regards to synaptic plasticity; regulating synaptic functional strength, including long-term potentiation which is an experience-dependent process important for learning and memory (Squire et al., 2008).

\section{BDNF}

BDNF is the commonest NT regulating neural plasticity, particularly in the limbic system (Kim et al., 2013). BDNF supports neurite growth by altering $\mathrm{Ca}^{2+}$ signalling in respective growth cones while increased synaptic concentrations lead to greater local elaboration of synaptic endings, additional dendritic growth and increased connectivity between pre- and postsynaptic knobs (Cunha et al., 2010). BDNF has been correlated with several psychiatric disorders, including; affective disorders, schizophrenia, Alzheimer's disease and substance misuse [9]; suggesting a pleiotropic action of the BDNF gene (Alexopoulos et al., 2010). An ongoing body of research is attempting to understand the implications BDNF has in; hippocampal volume shrinkage (Andrews et al., 2015), allelic variations 
(Kim et al., 2012), gene-environment interactions (Bozzini et al., 2009; Kim et al., 2013) and epigenetics (Lockwood et al., 2015).

\subsection{Depression}

The BDNF gene has several polymorphic markers, amongst which a single nucleotide polymorphism (SNP) at nucleotide 196G/A resulting in the substitution of a valine into a methionine nucleotide at codon 66 (Val66Met). This SNP effects intracellular processing and secretion, decreased activity-dependent secretion of BDNF and increased depression (Kim et al., 2013; Vinberg et al., 2013). Cytosine-guanine dinucleotides are the most stable and representative component of broader epigenetic modification and the methylation of these dinucleotides in promoter regions on the BDNF gene results in decreased BDNF synthesis (Kim et al., 2013).

Blood BDNF levels have been widely studied in individuals with unipolar depression, identifying low serum and plasma BDNF levels in MDD (Vinberg et al., 2013). A study demonstrated that serum BDNF levels decreased during depressive episodes and normalised in remission (Molendijk et al., 2011). Studies investigating the impact of BDNF Val66Met SNP has on serum and blood BDNF changes in cohorts of psychiatric patients did not reveal any significant association (Elfving et al., 2012; Terracciano et al., 2013), suggesting that the SNP is not associated with blood BDNF levels. Literature findings have been replicated when individuals with first onset of depression had lower blood BDNF levels than healthy participants with no relatives diagnosed with MDD, but no correlation have been found between severity and BDNF levels (Millas et al., 2011; Vinberg et al., 2013). Due to confounding results of the impact the Val66Met SNP has on peripheral BDNF, further research is necessary.

The relationship between serum BDNF levels and antidepressant treatment outcome in MDD (Mikoteit et al., 2014). Mikoteit et al., (2014) was investigated over six weeks, by assessing psychopathology severity, serum BDNF and predicted treatment outcome based on a combination of changes in serum BDNF. High baseline serum BDNF and early improvement to treatment predicted successful outcome while initial increase in serum BDNF during treatment was considered unsuccessful. Diverging from previous research, more severe depressive symptoms were reported by participants with high serum BDNF; the authors argued that this resulted from rigorous statistical procedures which excluded confounding factors with regards to age, gender, education and illness. However, issues like smoking and quality of sleep which alter serum BDNF were not accounted for. The authors concluded that pronounced serum BDNF in severe MDD due to Chronic Mild Stress (CMS) prior to treatment is probably a compensatory mechanism. Supporting this hypothesis is the fact that serum BDNF was not reported to increase upon remission of depressive symptoms (Mikoteit et al., 2014).

In another study serum BDNF levels were not reported to stabilise following antidepressant therapy. This deviation was associated to the fact that unlike 
other research, only one antidepressants was used, while reported that increases in serum BDNF is less likely to occur with Serotonin Norepinephrine Reuptake Inhibitors (SNRIs) (used in the study) than with SSRIs (Mikoteit et al., 2014).

Since BDNF crosses the blood brain barrier it is assumed that blood BDNF levels equal brain BDNF; for this reason, several studies tried to clarify the correlation between BDNF levels, MDD, ethnical groups, age and gender. However, no significant results have been reported (Kreinin et al., 2015). Research demonstrated a discrepancy in the lifetime prevalence of MDD between men and women of $11 \%$ and $21 \%$ respectively, indicating a correlation between gender, BDNF levels and MDD. Kreinin et al. (2015), investigated the diagnostic value of BDNF as a potential marker for MDD but no significance was reported.

Stratified analysis correlating BDNF levels and depression severity demonstrated insignificant differences in mild depression and significant differences in severely depressed participants. Upon gender discrimination, a significant positive correlation between blood BDNF and MDD severity has been shown in females but not in males (Kreinin et al., 2015). In agreement with other studies, low BDNF levels have been identified in untreated individuals diagnosed with MDD which increased upon antidepressant administration with no significant differences between SSRIs and SNRIs (Kreinin et al., 2015).

Depression is associated with decreased neural plasticity, diminished neuronal survival and atrophy mediated by hippocampal BDNF deficit due to chronic stress (Ye et al., 2011). Ye et al., (2011) investigated the correlation between BDNF infusions and hippocampal astrocytic plasticity in rats exhibiting depressive-like symptoms. Following three weeks of CMS, sucrose consumption, total travel distance and velocity significantly decreased when compared to the control group; implying that the group exposed to CMS exhibited depressive symptoms. The cohort was divided into three groups; control + saline, CMS + saline and CMS + BDNF. Upon analysis, significant differences following BDNF treatment were identified. Depressive symptoms were significantly increased in the CMS + saline when compared to the control + saline group. Depressive symptoms in the CMS + BDNF were significantly decreased when compared to the CMS + saline group, while no significant differences were identified between the control + saline and CMS + BDNF groups. The same pattern was also observed with regards to hippocampal astrocytic plasticity (Ye et al., 2011). These results echoed previous literature which demonstrated the positive link between BDNF and sucrose performance test (Larsen et al., 2010) and forced swimming test (Sirianni et al., 2010). Suggesting that hippocampal plasticity, CMS and MDD could be targeted by BDNF infusion (Ye et al., 2011) (Figure 1 and Figure 2).

Research suggests the possible correlation between Huntington's Disease and decreased BDNF levels. Huntington's Disease causes neurodegeneration while BDNF exerts neurogenic properties. SSRI administration improves BDNF levels thus increasing neurogenesis (Peng et al., 2008), slowing the disease progression. In a study conducted by Peng et al., (2008) sertraline was investigated for the 


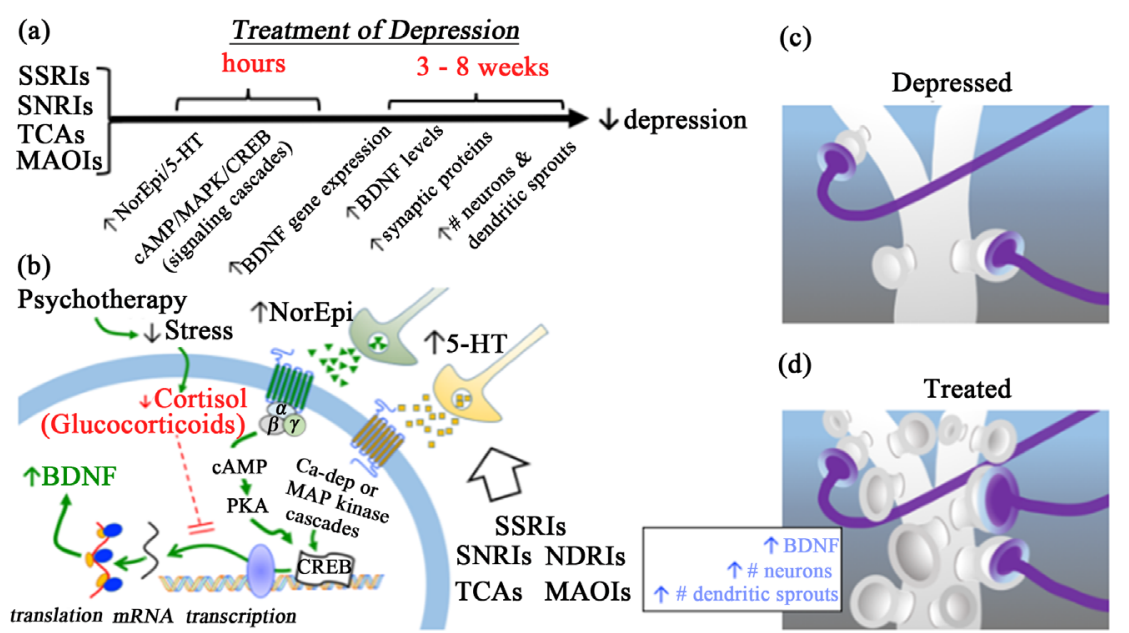

(Tulane University, School of Medicine, 2017)

Figure 1. BDNF: Normal vs Depressed.

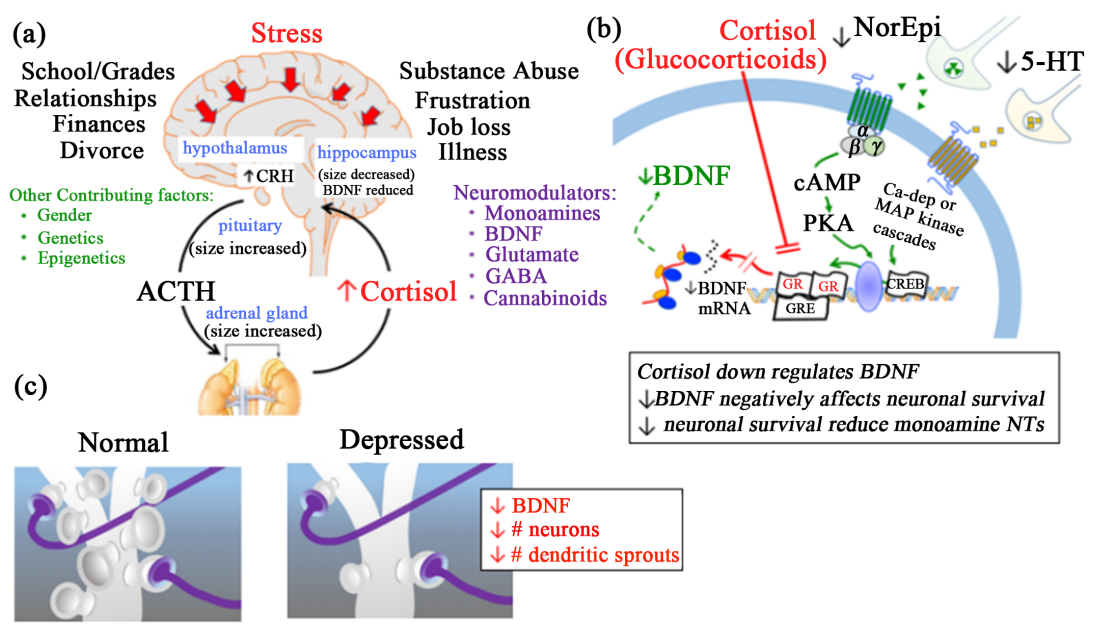

(Tulane University, School of Medicine, 2017)

Figure 2. BDNF: Depressed vs Treated.

improved survival, performance and amelioration of neuronal atrophy in mice with Huntington's Disease. Chronic administration of sertraline was reported to increase BDNF and serotonin expression, stimulating neurogenesis in rodents' brains (Peng et al., 2008). Sertraline increased neuronal survival, improved impaired motor function, reduced brain atrophy and increased neurogenesis particularly in the hippocampus. Despite the study was conducted in view of Huntington's Disease in rodents, these findings can be associated with increased neurogenesis, BDNF and serotonin levels achieved in human antidepressant treatment in MDD as reported by Umene-Nakano et al., (2010). Concluding that sertraline has neuro-protective properties, enhances serotonergic neurons and BDNF (Umene-Nakano et al., 2010) resulting in the respective neurological correlations each have on each other, the brain and neurotransmitter systems. Along with sertraline, paroxetine has also been shown to increase BDNF levels in 
mice (Peng et al., 2008) reinforcing the assumption that BDNF and serotonin have reciprocal stimulatory effects.

Despite that it is known that BDNF levels increase upon administering antidepressant medication, it is not clear how BDNF responds to different classes of antidepressants (Matrisciano et al., 2009). A recent study explored serum BDNF levels in patients with MDD treated with sertraline, escitalopram or venlafaxine and compared them to healthy control individuals. The study described a significant increase in BDNF after 5 weeks treatment with sertraline and stabilised by 6 months, an increase in BDNF with venlafaxine administration after 6 months while no significant results were obtained with escitalopram (Matrisciano et al., 2009). These results of resonate with other research conducted in rodents.

Late-life MDD is frequently accompanied with white matter abnormalities which may increase one's predisposition to MDD (Alexopoulos et al., 2010). Since BDNF regulates neuronal survival and synaptic plasticity, it may also have a role in white matter integrity and MDD in the elderly. Alexopoulos et al. (2010), investigated the effect Val66Met SNP has on remission from late-life MDD and explored the correlation with microstructural white matter abnormalities. The study reported that like younger individuals, depressed elderly Caucasian individuals with $\mathrm{BDNF}_{\mathrm{met}}$ allele were more likely to achieve remission than $\mathrm{BDNF}_{\text {val/val }}$ homozygotes after 12 weeks of treatment. Additionally, microstructural abnormalities in the corpus callosum, left superior corona radiata and right inferior longitudinal fasciculus were associated with lower remission rates, but no correlation has been found between the studied BDNF allele, elderly MDD and white matter abnormalities (Alexopoulos et al., 2010).

Depression is also common after stroke, both psychosocial and biological factors are important in the development of post-stroke depression (PSD) (Kim et al., 2013). BDNF expression is regulated by epigenetic chromatin remodelling in the gene promoter regions. Previous depression was described in $15 \%$ of the participants. The PSD group was more likely to have had a history of depression when compared to those without PSD. Results showed that BDNF promoter methylation at $\mathrm{CpG}$ site 6 exhibited higher incidence of prevalent, persistent and incident PSD while methylation at CpG sites 1,2 and 4 were more associated with incident PSD. Principally, the higher the methylation of BDNF promoter gene the higher the risk of prevalent, persistent and incident PSD with significant worsening of symptoms after one year (Kim et al., 2013). No methylation-genotype interactions were identified (Kim et al., 2013).

Stress has been shown to reduce BDNF-mediated signalling in the hippocampus resulting in depressive symptoms in animal studies. Human studies linked BDNF Val66Met SNP with reduced activity-dependant BDNF secretion and decreased hippocampal volume, however genetic studies did not identify any association with depression (Kim et al., 2013). Suggesting that genetic vulnerability might play a stronger role when combined with specific environmental stressors known to have a substantial effect on risk of depression-gene-environment in- 
teraction. This thesis echoed in recent studies which identified a link between genetic vulnerability and stressors. The Val66Met allele has also been linked to depression comorbid to; coronary artery disease (Bozzini et al., 2009), stroke (Kim et al., 2012) and breast cancer surgery (Kim et al., 2012). The association between stroke, BDNF and depression has been made since;

1) BDNF relies on activity-dependant release mechanism which is decreased with higher methylation (thus in stroke and increased methylation, BDNF is decreased).

2) Since BDNF has antidepressant activity, lower BDNF secretion after stroke increases depressive symptoms.

(Kim et al., 2013)

Another study investigating PSD investigated the independent and interactive association of serotonin transporter, serotonin 2a receptor and BDNF genes in PSD (Kim et al., 2012). A total of 276 participants with a mean age of 64.3 years were recruited and interviewed at 12 days post-stroke. $10.5 \%$ were diagnosed with MDD, $17.4 \%$ with minor depression, 5.4\% reported previous depression and $27.9 \%$ were categorised as having PSD. The PSD group was more likely to be older, have experienced past depressive episodes and/or stroke and more likely to have had an anterior stroke. Genotyping demonstrated that participants with any form of depression had significantly higher short allele levels in the promoter region of the serotonin transporter, $1438 \mathrm{~A}$ allele in the serotonin receptor 2a gene and $\mathrm{BDNF}_{\text {met }}$ allele (Kim et al., 2012).

The $\mathrm{s} / \mathrm{s}$ allele in the serotonin transporter and the $\mathrm{BDNF}_{\text {met/met }}$ genotypes were significantly associated with any depression and only the $1438 \mathrm{~A} / \mathrm{A}$ serotonin receptor gene was linked to MDD. Serotonin transporter and receptor distribution were not independent; however, no significant interactive effects of genes have been identified with stroke location. This is the only study found investigating the correlation between serotonin receptor 2a polymorphisms and PSD (Kim et al., 2012). Asserting the study's reliability is the fact that despite the small number of participants recruited, depression was ascertained prior recruitment using a structured diagnostic interview based on the Diagnostic Statistical Manual IV (DSM-IV) at a similar time-point in all participants.

The role of epigenetics in the occurrence of depression and suicide has also been researched and a systematic review conducted by Lockwood et al. (2015), showed that a correlation between these factors exists. If the link between environmental factors and depression is established, thus suicide prevention programs could be improved accordingly (Lockwood et al., 2015). Most of the papers reviewed identified a link between epigenetics, depression and suicide. Hyper-methylation in TrkB T1 region was found in subjects who died by suicide in comparison to control subjects (Ernst et al., 2009; Maussion et al., 2014), however another study found no differences in the TrkB T1 region between suicide and non-suicide groups (Keller et al., 2011). Ernst et al., (2009) and Maussion et al., (2014) looked at several brain regions including Brodmann's areas 8 and 9 while Keller et al., (2011) looked at the Wernicke's area; thus, possibly the me- 
thylation changed are not observed concurrently in all brain regions. Ernst et al., (2009) and Maussion et al., (2014) were also conducted by the same laboratory, thus the possibility that both had overlapping participants cannot be excluded (Lockwood et al., 2015).

BDNF gene methylation was also found to be significantly associated with MDD and suicidal ideation. BDNF methylation was more pronounced in individuals with MDD than participants with manic or bipolar disorder. The study also reported that lithium carbonate and sodium valproate decrease the level of BDNF methylation, however statistical significance was not reached (Dell; Osso et al., 2014). In this review the authors acknowledged that the studies utilised were all preliminary studies with small samples, however most studies agreed with each other, confirming the correlation between epigenetic alterations, MDD and suicide (Lockwood et al., 2015).

\subsection{Suicide}

Suicidal behaviour is generally associated as part of the symptomatology of a psychiatric disorder. Despite that it is strongly associated with psychiatric co-morbidities, recent research also considered suicide as an independent phenomenon with a high percentage of heritability (Sears et al., 2013). Supporting this concept is research which demonstrated that children of suicide attempters are six times more susceptible to attempt suicide than children of non-suicide attempters, even when diagnosed with a psychiatric illness (Brent et al., 2002). Higher Cholecystokinin (CCK) receptors in frontal cortex and Cholecystokinin B Receptor (CCKBR) in the cerebellum, pre-frontal cortex and cingulate gyrus have been reported in individuals who died by suicide. Considering these facts, a study tried to identify any possible correlation between SNPs in the BDNF, CCK and CCKBR genes and suicide. From the BDNF gene, five SNPs were significantly correlated with attempted suicide, however no association was identified between Val66Met allele and attempted suicide; which replicated results from other studies which further elaborated that the Val66Met allele is significantly associated with MDD but not with attempted suicide (Neves et al., 2011). CCK and CCKBR have been previously studied and relationship with a number of psychiatric disorders has been established. 12 CCKBR SNPs were significantly associated with attempted suicide. No CCK SNPs were found to be associated with suicide. Despite significant associations of the respective genes with attempted suicide, none of the associations have evidence supporting their association with suicidality in genome-wide association studies.

Low serum BDNF levels are generally described in MDD patients; however research reports conflicting results with regards serum BDNF levels in completed suicides. A study conducted amongst 281 participants (84 attempted suicides, 104 psychiatric controls and 93 community controls) reported no significant differences amongst the different groups after adjustment to age, sex, age, psychiatric co-morbidity and smoking between attempted suicide and serum BDNF levels (Eisen et al., 2016). 
Suicidal behaviours are interactively influenced by biological, psychiatric, psychosocial, interpersonal, cultural factors and neurotransmitters. The BDNF promoter gene is exposed to epigenetic modification such as $\mathrm{CpG}$ methylation which decreases BDNF synthesis and expression (Kang et al., 2013). Studies have shown that suicidal behaviours increase with a gene-specific methylation of the BDNF promoter, irrespective of genome-wide methylation level (Keller et al., 2010). Thus, Kang et al., (2013) investigated the correlation between BDNF promoter hyper-methylation and suicidal behaviour in a MDD group receiving antidepressant medication for 12 weeks. From the recruited sample; $19.4 \%$ previously attempted suicide, $51.9 \%$ had suicidal ideations prior to the study, $52.8 \%$ had suicidal ideations during the study and $25 \%$ newly developed suicidal ideations during the study. The high percentage of new participants reporting suicidal ideations has been accounted for by the authors since also ruminating thoughts of suicide without actual plans have been considered as suicidal ideations (Kang et al., 2013). The authors should have differentiated between passively and actively suicidal, further differentiating and focusing their research on the respective targeted population. Higher degrees of BDNF methylation were significantly associated with males; this may be the causation of more aggressive and thus completed suicides in males. Previous suicidal attempts and suicidal ideation during treatment were associated with higher BDNF methylation but no link has been reported between type of antidepressant and suicidality (Kang et al., 2013).

Other studies demonstrated a relationship between BDNF methylation and completed suicides (Keller et al., 2010), however in these studies post-mortem brain tissues have been utilised; thus, not considering the length of exposure between time of exposure to the methylated gene, outcome and possible epigenetic modification after death (Kang et al., 2013). The authors presumed that $\mathrm{BDNF}$ is decreased in individuals with higher methylation, thus could have also analysed blood BDNF levels to confirm this assumption.

Previously conducted studies demonstrated that the BDNF Val66Met SNP is not only correlated to MDD, but is also associated to suicidal behaviour in MDD, suicide attempts in bipolar disorder and schizophrenia (Ratta-apha et al., 2013). A study that attempted to replicate potential associations between SNPs on the BDNF gene and suicide demonstrated that when compared to the $\mathrm{BDNF}_{\mathrm{val}}$ allele, the $\mathrm{BDNF}_{\text {met }}$ allele was found to be associated with suicide attempts in Asian populations but not with completed suicide. No significant differences were reported between completed suicide and control groups in $\mathrm{BDNF}_{\mathrm{val}}$ and $\mathrm{BDNF}_{\mathrm{met}}$ allele occurrence (Pregelj et al., 2011; Ratta-apha et al., 2013). The authors speculate that this discrepancy may result due to the pathogenesis of completed suicides being more complex than that of attempted suicides.

The $\mathrm{BDNF}_{\text {met }}$ allele was found to have no correlation to suicide attempts with regards to age, sex, ethnicity, marital status, schooling years, familial psychiatric history and substance misuse. However, the presence of the $\mathrm{BDNF}_{\text {met }}$ allele was 
correlated with high lethality attempted suicides. As predicted, male gender was also independently and significantly associated with lethality of attempted suicide. Additionally, suicide lethality has also been correlated with decreased serotonergic transmission (Schenkel et al., 2010).

It has been reported that genes encoding for serotonergic, dopaminergic, noradrenergic, GABAergic, NTs and the HPA axis make up $55 \%$ of the genetic heritable contribution of suicide. Allelic variations effecting BDNF regulation impact the hereabove mentioned processes (Ropret et al., 2015). Studies investigating BDNF allelic variations and suicide mainly focused on Val66Met SNP; a study conducted by Ropret et al., (2015) investigated the effect seven other SNPs in the BDNF regulatory gene have on suicidal behaviour. Analyses of allele and genotype frequency between control and study groups, even after adjustment for age, gender and alcohol dependence did not reveal significant associations between the studied SNPs and suicide. However, haplotypic analysis associated the haplotype C-A-T-T-C with completed suicide. A T to C base change in the first position of the haplotype located in a potential microRNA binding site of rs7124442 minor allele was found to be significantly more abundant in individuals who died by suicide than the other SNPs. Concluding that the rs7124442 minor allele plays a phenotypic role in suicide. Haplotypic analysis adjusted for confounding factors (age, gender and alcohol dependence) supported this proposed concept (Ropret et al., 2015). Nevertheless, since a relatively small sample has been recruited, further research with larger bodies of participants is necessary.

The literature describes that apart from hyper-methylation of the BDNF gene, hyper-methylation of TrkB gene in the frontal cortex and hippocampus is also associated with completed suicides. A recent study compared the epigenetic modifications of TrkB gene occurring in the Wernicke's area in a sample of 18 suicide subjects with 18 control subjects. The study reported no significant association between TrkB expression and promoter methylation to completed suicides, but significant hyper-methylation of the BDNF promoter gene identified in other studies (Keller et al., 2010) was replicated (Keller et al., 2011). The results in this study cannot be considered as conclusive and exhaustive since a sample of only 18 participants has been recruited.

\section{Discussion}

The increased evidence of different processes undergoing in programmed cell death to develop pharmacological agents to treat pathological neuronal death, is now better defined by the widely held assumption that neuronal death is the primary culprit in the pathophysiology of these diseases. Increasing evidence suggests that neuronal and synaptic dysfunctions precede neuronal degeneration identified as the contributing factor for the behavioural pathology in neurodegenerative diseases, and to a certain extent, also MDD (Squire et al., 2008).

Hyper-methylation of the BDNF promoter gene due to epigenetic factors has 
been significantly correlated to MDD. Since most individuals who die by suicide are diagnosed with MDD; important causative factors in the environment and biomarkers (hyper-methylation of BDNF promoter gene) have been identified to increase the risk and cause suicide. This newly developed knowledge might help to move toward precision medicine and develop improved suicide programs adequately targeting vulnerable populations. Additionally, since epigenetic modifications result in functional modifications while conserving the Watson and Crick model of DNA, epigenetic modifications possibly offer an easier and more effective method for diagnosis and treatment methods (Lockwood et al., 2015). Nonetheless further studies are necessary to identify and manage functional modification of genes and target therapeutic agents. Since suicidal ideation is commonly described in other psychiatric disorders (Kang et al., 2013) further research should investigate the relationship between methylation and other psychiatric disorders leading to suicide. Additionally, since DNA methylation is potentially reversible according to surrounding environmental factors, further research is necessary to identify pharmacological agents which reverse methylation.

Controversially, despite suicidal ideation is a behaviour commonly associated with other psychiatric disorders, recent research considered suicide as a phenomenon with high heritability factors independently occurring from psychiatric diseases (Sears et al., 2013). In line with this model, a significant association has been made between the $\mathrm{BDNF}_{\text {met }}$ SNP and lethality of attempted suicide, with the authors stating that suicide is a multi-factorial phenomenon and apart BDNF other neurotransmitters, genetic, environmental and cultural factors might account for the different results encountered in the body of literature (Schenkel et al., 2010). Thus, suicide is generally associated with but not necessarily a result of psychiatric disease.

Nonetheless, independent of psychiatric diagnoses, abnormal BDNF function has been associated with elevated suicidality and possibly that treatment-induced improvement in BDNF system prevents or decreases suicidal behaviour independently of improvement in psychiatric symptomatology [9]. Studies utilising laboratory rats demonstrated that BDNF produced antidepressant results in depressed rats when; delivered as a continuous high-dose infusion to the mid-brain, one-time low-dose infusion to the hippocampus and ventricles and continuous high-dose infusion to the hippocampus (Ye et al., 2011). Apart from confirming the results in previous literature that a correlation exists between BDNF and MDD also demonstrated that the administration of BDNF reversed and effectively treated MDD. However, it is unclear how different BDNF dosing regimens would affect hippocampal astrocytic plasticity.

A correlation exists between sertraline administration and resultant increases in BDNF and serotonin in MDD. These correlations have been deducted from separate research conducted on laboratory rats and humans which investigated similar factors and achieved increased serum BDNF levels. Despite that the 
study conducted on laboratory rats investigated increases in serum BDNF levels in Huntington's Disease, increases in BDNF levels in both Huntington's Disease and MDD result in increased neuronal survival, neurogenesis and decreased brain atrophy (Peng et al., 2008). Similar results have been reported in human studies, describing an increase in serotonergic neurons and BNDF and their interactive relationship (Umene-Nakano et al., 2010). Additionally, sertraline and venlafaxine have been identified to increase BDNF levels while escitalopram does not (Matrisciano et al., 2009).

Due to the trophic effect of BDNF on neurons, alterations in BDNF levels might lead to brain atrophy and neurodegenerative and neuropsychiatric disorders. Another theory suggests that BDNF and serotonin interact together; serotonin receptors stimulate BDNF synthesis and secretion while BDNF stimulated serotonin neural axon plasticity and serotonergic transmission. Thus, explaining the implication of serotonin in the pathophysiology of MDD and suicide. Concluding that the $\mathrm{BDNF}_{\text {met }}$ allele alters BDNF synthesis and secretion which in turn down-regulated serotonin function, resulting in suicidal behaviour and increased lethality in attempted suicides (Schenkel et al., 2010).

Several studies have been conducted reporting significant associations between serum BDNF levels and suicide; however these studies generally recruited a limited number of participants. Eisen et al., (2016) who reportedly recruited one of the largest samples investigating this issue, reported no significant correlation between serum BDNF levels and suicide. However, due to the confounding existing body of literature, absence of studies high in the hierarchy of evidence replicating similar results, further research investigating this correlation and recruiting larger samples is necessary.

The lack of association between all BDNF SNPs (except Val66Met allele) with attempted suicide suggests that BDNF has no or little role in the aetiology of suicide; no evidence assessing genome-wide association studies exists associating BDNF to suicide. Despite significant associations of the respective genes with attempted suicide, none of the associations have evidence supporting their association with suicidality in genome-wide association studies (Sears et al., 2013). Confounding results are still being reported, which demonstrate opposing implications in its impact on suicide. Well controlled studies controlling all possible contributing factors which may interfere with BDNF levels should be conducted. Research investigating the effect of rarer genetic variants with prominent functional impact in the BDNF gene should also be studied and explore their impact on different ethnic groups. A factor making the study of this phenomenon more difficult is to separate genetic risk for suicide from genetic risk for psychiatric disorders. This difficulty is sometimes tackled by recruiting suicidal (test) individuals and control individuals (non-suicidal) suffering from the same psychiatric conditions (Sears et al., 2013). Since epigenetic changes are potentially reversed by pharmacological agents (Keller et al., 2011), the identification of specific epigenetic modifications on different genes in different brain areas 
would assists to target the necessary treatment and improve the remission rates to specific diseases. Thus, research obtaining a "map" of any methylation processes and expression of depression and suicide related genes in different brain areas is necessary to identify depression and suicide specific epigenetic brain variations.

\section{Conclusion}

The absolute aetiology of MDD is unknown; however tenets for further research have been clearly identified. Influencing genetic, environmental and cultural factors leading towards the development of MDD and suicide have been identified but further research is necessary to identify how these interact together and cause the resulting pathophysiology and behavioural changes in psychiatric disease.

\section{Dedication}

Dedicated to J.S., a colleague but most of all a loyal friend who unfortunately died due to depression. May the experience of your suffering remind us that as professionals we are not immune to mental health issues and help us to better assist those in need. Carved in the literature and concealed with an immortal veil of surrealism, your memory shall never be forgotten.

\section{Funding}

This research has received no specific grant from any funding agency in the public, commercial or non-profit sectors.

\section{Conflicts of Interest}

The authors report no conflicts of interest.

\section{References}

[1] WHO (2016) World Health Organisation. http://www.who.int/mediacentre/factsheets/fs369/en/

[2] Videbeck, S.L. (2008) Psychiatric-Mental Health Nursing. 4th Edition, Lippincott Williams \& Wilkins, Philadelphia.

[3] Richards, C.S. and O’Hara, M.W. (2014) The Oxford Handbook of Depression and Comorbidity. Oxford University Press, Oxford. https://doi.org/10.1093/oxfordhb/9780199797004.001.0001

[4] Davies, T. and Craig, T. (2009) ABC of Mental Health. 2nd Edition, Wiley-Blackwell, Hoboken.

[5] OECD (2014) European Commission-Public Health. http://ec.europa.eu/health/state/docs/health_glance_2014_en.pdf

[6] World Health Organization (2014) Preventing Suicide-A Global Imperative.

[7] CDC. (2015) Centers for Disease Control and Prevention. http://www.cdc.gov/violenceprevention/pdf/suicide-datasheet-a.PDF

[8] Eurostat (2016) Statistics Explained. 
https://ec.europa.eu/eurostat/statistics-explained/index.php?title=Health

[9] Sher L. (2011) Brain-Derived Neurotrophic Factor and Suicidal Behavior. QJM: An International Journal of Medicine, 104, 455-458. 